\title{
Transatlantica
}

Revue d'études américaines. American Studies Journal

\section{Sally Mann, La chair toute nue}

\section{Lisa Rossi}

\section{(2) OpenEdition}

Journals

Édition électronique

URL : https://journals.openedition.org/transatlantica/4684

DOI : $10.4000 /$ transatlantica.4684

ISSN : 1765-2766

\section{Éditeur}

Association française d'Etudes Américaines (AFEA)

\section{Référence électronique}

Lisa Rossi, « Sally Mann, La chair toute nue », Transatlantica [En ligne], 2 | 2009, mis en ligne le 01 février 2010, consulté le 31 janvier 2023. URL : http://journals.openedition.org/transatlantica/4684 ; DOI : https://doi.org/10.4000/transatlantica.4684

Ce document a été généré automatiquement le 31 janvier 2023.

\section{(c) (i) (9)}

Creative Commons - Attribution - Pas d'Utilisation Commerciale - Pas de Modification 4.0 International - CC BY-NC-ND 4.0

https://creativecommons.org/licenses/by-nc-nd/4.0/ 


\title{
Sally Mann, La chair toute nue
}

\author{
Lisa Rossi
}

1 Il y a trente ans de cela, la féministe Margaret Walters remarquait qu'il subsistait une distinction tenace entre « le Sexe-qui-regarde » et le "Sexe-qui-est-regardé ${ }^{1}$ » dans les conventions de la représentation du nu. Une vingtaine d'années plus tard, Germaine Greer réaffirmait cette assertion et déplorait que toute transgression de cet état de fait provoquât la controverse, ou pire, n'éveillât aucun intérêt (The Beautiful Boy, 2003). Si le mouvement féministe des années 1970 a vu des artistes - femmes, majoritairement s'intéresser à ce sujet, parmi lesquelles Deborah Law, Sylvia Sleigh (Philip Golub Reclining, 1971) ou plus tardivement les "Guerilla Girls», il semble que leurs réclamations n'aient fait que renforcer cette dichotomie : plus ou moins humoristiques ou frondeuses, elles persistaient à s'adresser à un public masculin, probablement occidental et hétérosexuel. Cette conception du nu est de fait aujourd'hui encore prégnante et il semble qu'elle le soit d'autant plus en photographie. Si l'on peut facilement citer une dizaine de photographes ayant à un moment ou un autre de leurs carrières choisi d'explorer le nu féminin, il devient franchement plus ardu de trouver parmi leurs consœurs la démarche inverse. On peut bien entendu arguer que Nan Goldin, ou avant elle Diane Arbus, ont porté leurs regards sur des hommes, souvent nus ou légèrement vêtus. Mais leur statut de freaks, de marginaux - parce qu'homosexuels, travestis, et/ou obèses - les habillait plus sûrement que n'importe quel vêtement. En d'autres termes, leur représentation n'avait pas pour objet d'exprimer, voire de satisfaire un possible plaisir visuel féminin. Dans le domaine de l'art, l'homme-objet de désir ou de célébration s'est finalement vu majoritairement honorer dans la catégorie restreinte de la photographie contemporaine, elle-même parfois récriée, de l'homoérotisme - de Mapplethorpe à John Dugdale ou au couple McDermott et McGough. Dans ce contexte, il est notable que la Gagosian Gallery de New York ait choisi d'exposer sur ses murs prestigieux, du 15 septembre au 31 octobre 2009, la dernière série de la photographe américaine Sally Mann, intitulée Proud Flesh et exclusivement composée de nus masculins.

2 Sally Mann a conquis sa renommée dans un climat de controverse liée à sa représentation d'une enfance sensuelle et grave, dont les protagonistes étaient ses propres enfants et la toile de fond, un ancestral paysage virginien. La nudité d'Emmett, 
Jesse et Virginia (et les accusations d'inceste ou d'incitation à la pédophilie qui en ont découlé), était l'une des causes du scandale, tout comme l'apparente immoralité avec laquelle la photographe semblait vendre leur intimité. Plus que tout, Immediate Family bouleversait les codes sacrés d'une enfance idéalisée où le sexe, le danger, la violence, la décrépitude ou la mort n'existent pas. Dans cette série, l'artiste posait néanmoins les jalons d'une représentation masculine doucement subversive. Car si on retrouvait Emmett dans la position du jeune garçon vulnérable et abandonné, fidèle à des canons hérités de la Renaissance, l'objectif de sa mère révélait aussi sa force et sa virilité en devenir, une assurance tranquille et éhontée face aux regards inquisiteurs des spectateurs (The Last Time Emmett Modeled Nude, 1987). On pouvait surtout apercevoir, par intermittence, la figure de Larry Mann, époux de l'artiste et père des trois enfants modèles. On le voyait embrasser ses enfants, les prendre dans ses bras, les consoler, tour à tour en père affectueux, nourrice, et même madone, si l'on se réfère à la composition de The Good Father (1990) - les deux filles de l'artiste lovées contre leur père assis - qui rappelle en tout point celle de La Vierge au Chardonneret de Raphael (1505-1506). Sally Mann le donnait aussi à voir, plus rarement, dans des mises en scène érotiques, parfois teintées d'humour, comme dans le cliché où, les pieds dans le lit de la rivière, nu, dans une pose classique de contrapposto, il tenait contre son corps, à hauteur de son bassin, une cane à pêche dressée vers les cieux (Larry, 1977). Connaissant la prédilection de la photographe pour l'intime et l'immédiat, il n'est pas surprenant que Proud Flesh soit entièrement consacrée au portrait de celui qui partage sa vie depuis quarante ans.

3 La démarche n'est pas nouvelle. Avant Sally Mann, des hommes, parmi lesquels Emmett Gowin et Harry Callahan, ont fait de leurs épouses respectives les points d'ancrage de leurs œuvres photographiques. De la même façon, Sally Mann a photographié son mari et leur vie commune depuis le premier jour de leur rencontre dans une série familièrement baptisée Marital Trust. Prolongement de cette série, Proud Flesh résulte néanmoins d'un évènement décisif dans la vie du couple. Il y a une dizaine d'années, on a diagnostiqué chez Larry Mann une forme de dystrophie musculaire, une malade évolutive, lente mais irréversible. Le titre de la série est d'ailleurs sujet à une double interprétation, car si "proud flesh" se traduit littéralement par "chair fière ${ }^{2}$ ", l'expression est également un terme utilisé en médecine - en particulier vétérinaire et équine -, pour désigner le surplus de tissus de granulation qui peut se former sur une plaie durant le processus de cicatrisation, avant qu'une peau nouvelle ne recouvre la blessure. Nul doute que la cavalière qu'est Sally Mann ait connaissance de ce phénomène qui, non traité, ralentit le processus de guérison et inflige des déformations anatomiques à un organisme par ailleurs sain. C'est ce même paradoxe, celui de la vigueur malgré la maladie, et, inversement, de la maladie malgré la vigueur, qui se devine dans les propos de la photographe lorsqu'elle affirme s'étonner encore à la vue des membres amoindris de son mari ${ }^{3}$, bien qu'elle se soit appliquée pendant plusieurs années à étudier son corps avec minutie. Que le désir de figer la maladie dans le collodion ou que le besoin de ré-apprivoiser un corps qui devenait étranger président à cette démarche importe peu. Dans chacune des trente-trois photographies qui composent l'exposition, Larry Mann prend la pose, son corps nu tantôt nimbé de lumière tantôt dévoré par l'obscurité, altéré par les nébulosités de l'émulsion photographique ou au contraire paré de motifs métalliques, semblable à un dieu de la mythologie grecque. Et de fait, l'artiste ne manque pas de se référer, notamment par le biais de ses titres, aux canons esthétiques de la Grèce ancienne, telle une Psyché osant 
faire le jour sur le visage de son amant. Hephaestus (Fig. 1) est probablement le cliché le plus emblématique de la série : debout devant une table qui l'isole du regardeur, coupé à hauteur du visage, Larry Mann donne à voir ses larges épaules, son torse robuste, tandis que la lumière caresse un bras solide pour laisser dans l'ombre celui qui s'amincit inexorablement. Une longue érosion argentique parcourt son corps du cou jusqu'à l'aine. "It's named after a Greek god - an easy choice, since Larry was a blacksmith, too, and is also lame. He looks so godlike, yet metallic and shiny ${ }^{4}$ » a déclaré l'artiste. Larry Mann n'est pas un chérubin, ni un adolescent alangui, encore moins l'objet soumis d'un quelconque désir sexuel. C'est un homme d'âge mûr, conscient, offert mais non assujetti, amoindri mais toujours puissant, sur lequel l'artiste pose son regard singulier, à la fois passionné et cru. "And I look with the passions of both eye and heart, but in that ardent heart, there must also be a splinter of ice » reconnait-elle dans le communiqué de presse de l'exposition ${ }^{5}$. "It is a testament to Larry's tremendous dignity and strength that he allowed me to take the pictures that I did $^{6}{ }^{\prime}$.

En effet, rien n'est simplement joli ou sentimental dans l'œuvre de Sally Mann. La maladie de Larry n'est pas le sujet de la série, mais elle la parcourt avec une persistance de louve affamée. La violence du sentiment affleure à la surface des photographies. La mort attend son heure larvée dans l'ombre, et il est significatif que le seul portrait qu'elle ait fait du visage de son époux le représente couché, les yeux fermés comme pour un dernier sommeil, dans une pose qui rappelle inévitablement les clichés postmortem de l'ère victorienne (Was Ever Love, Fig. 2). Le corps même de l'être aimé est abîmé, tâché, déformé, comme sauvé in extremis des flammes. Dans The Ponder Heart (Fig. 3), le dos de Larry Mann est griffé, ou plutôt, l'image du dos de Larry Mann est griffée, comme si une main trop avide avait cherché à le saisir à travers le médium photographique ou en avait usé la surface à trop la caresser. Les clichés de Sally Mann sont des fétiches, mais aussi des memento mori. Les images sont déjà nécrosées, déjà anciennes, déjà posthumes. C'est ici qu'intervient l'importance du médium photographique. Depuis ses premières utilisations du collodion humide sur plaque de verre dans les années 1990, l'artiste a investi ce procédé d'une valeur mémorielle aussi bien historique qu'idiosyncrasique, accentuée par un traitement pictorialiste de l'image. Chérissant les imperfections générées par sa pratique volontairement approximative du collodion, elle a livré une puissante mythification d'un sud longtemps déshérité en puisant dans son histoire tourmentée (Deep South, Bulfinch Press, 2005). Elle a fait de chacun de ses clichés de champs de bataille une icône des martyrs de la guerre de Sécession ${ }^{7}$. D’un centre médico-légal du Tennessee, familièrement nommé The Body Farm, où les cadavres sont laissés à leur décomposition, elle a tiré des allégories du cycle de la vie et de ce qui doit demeurer quand la matière

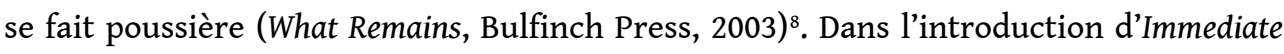
Family (Aperture, 1992), Sally Mann posait une question qui, plus de deux décennies plus tard, est toujours au cœur de son œuvre: « How is it that we must hold what we love tight to us, against our very bones, knowing we must also, when the time comes, let it go?». Il n'est pas étonnant, à cet égard, que le collodion, substance mise à contribution par Frederick Scott Archer pour sensibiliser les plaques de verre en 1851 et utilisée pour panser les blessés durant la Guerre Civile américaine, soit devenu le médium de prédilection de l'artiste. Le procédé au collodion de la photographe tour à tour coule dans sa matière visqueuse et ambrée ce qu'il faut sauver de la disparition et révèle les blessures que le temps inflige aux êtres vivants. C'est ainsi que Larry Mann se dresse dans toute sa splendeur d'homme vigoureux et indéniablement présent (Fig. 4), et 
l'instant d'après, glisse vers le même état de décrépitude organique que les cadavres tronqués de la Body Farm (Fig. 5). Un "éclat de glace»? Plutôt l'acuité d'une photographe qui refuse de vaciller devant la finitude de l'existence, afin de mieux embrasser la vie. Comme l'a écrit Philippe Ariès, «Plus grande est la passion de la vie, plus douloureuse l'amertume d'en être arraché» (Images de l'Homme devant la mort, 1983) : parler de la mort ne revient donc jamais qu'à parler de son attachement à la vie.

Bien que Proud Flesh partage une indiscutable parenté avec les précédents travaux de Sally Mann, il faut tout de même noter qu'elle s'en distingue à plusieurs égards. Cette série est probablement la plus personnelle et la plus dépouillée que l'artiste ait jamais réalisée. Une table, un banc, des draps élimés, un tabouret, un verre oublié, un tapis aux motifs démodés, le tout fondu dans les nébulosités du collodion, sont les seuls accessoires convoqués ici (Fig. 6). La photographe n'autorise aucune narration au sein de l'image. Les mises en scènes souvent élaborées d'Immediate Family visaient à incarner de grands thèmes: "We are spinning a story of what is to grow up... anger, love, death, sensuality, and beauty ${ }^{9}$ ». Les somptueux paysages de Deep South disaient l'immuabilité de la nature et réhabilitaient un Sud profond à la mélancolie teintée d'onirisme. What remains était une méditation sur la mort et la vanité de la vie traversée sans lien terrestre. Ce que Sally Mann nous livre ici, c'est la chair toute nue. Et pas n'importe laquelle, est-il besoin de le rappeler. De la même façon, elle a supprimé toute narration du dispositif muséographique. Là où les vers de Walt Whitman et de Bossuet donnaient corps au cheminement douloureux de l'exposition What remains, les murs de la Gagosian Gallery sont restés vierges de tout commentaire. Plus surprenant encore, le luxueux livre publié conjointement par Aperture et la Gagosian Gallery ${ }^{10}$ ne fait pour l'occasion aucune place à la prose pourtant précieuse de l'artiste et ne contient qu'un texte introductif de la poétesse américaine D.C. Wright ${ }^{11}$. Le format même des photographies $(38,1 \times 34,3 \mathrm{~cm})$ semble relever de l'intime et non plus du monumental comme c'était le cas pour les tirages iconiques de la série Faces ${ }^{12}(127$ x 101,6 cm). Pour la première fois, l'artiste laisse entrer le spectateur dans la quiétude de son atelier. Plus exactement, c'est la relation entre le photographe et son modèle qu'elle nous donne à voir, non plus Psyché mais Pygmalion. Au-delà de la transgression des codes de la photographie de nu, de l'enregistrement obsessionnel du déclin d'un être cher, ce retour aux fondements du portrait est peut-être la leçon la plus importante de la série, en même temps qu'elle jette une lumière nouvelle sur la controverse qui a suivi la publication d'Immediate Family: "Rhetorically circumnavigate it any way you will, but exploitation lies at the root of every interaction between photographer and subject, even forty years into it. Larry and I both understand how ethically complex and potent the act of making photographs is, how freighted with issues of honesty, responsibility, power and complicity, and how so many good images come at the expense of the sitter, in one way or another " annonce-telle dans le communiqué de presse, "These new images, we both knew, would come at his ${ }^{13}$ ». Oui, regarder ces photographies donne l'impression de lire des lettres d'amour qui ne nous sont pas destinées. Oui, les œuvres de Sally Mann peuvent être perçues comme autant de forfaits d'une exhibitionniste impénitente. Oui, Sally Mann est consciente du mélange d'innocence et de perversité que recèle chacune de ses photographies. Justement. Elle nous dit que la photographie est un médium vorace, qu'il exige du modèle un véritable don de soi, un acte d'amour, voire de dévotion. Et que cet acte, tout photographe a dû ou devrait le ressentir un jour au moment de capturer les traits d'un autre être humain, « is both heartbreaking and terrifying at once ${ }^{14}$ ». 
Pour autant, il ne faudrait pas se laisser prendre au piège que nous tend l'artiste depuis ses premières séries. Sa matière première est peut-être l'immédiat, plus que l'intime d'ailleurs, mais c'est un immédiat puissamment déformé par le prisme du mythe. Croire l'artiste sur parole reviendrait à nier son intelligence artistique. Les photographies de la série Immediate Family n'ont jamais été des snapshots mais des tableaux soigneusement composés à la façon des tableaux vivants du 19e siècle, comme en témoignent d'ailleurs les nombreuses citations qu'ils recèlent - de Dorothea Lange, Robert Frank, Edward Weston, William Eggleston, Manet, jusqu'à l'évocation de figures mythologiques (les Trois Grâces, la Gorgone). Sa persistance à inscrire son œuvre, et à travers elle sa propre généalogie, dans un Sud peuplé de figures excentriques dont elle se veut l'héritière, dans une tradition d'honneur et de nostalgie héritée de la guerre de Sécession, est aussi l'un des fils rouges de sa carrière ${ }^{15}$. L'exposition Proud Flesh, par son dépouillement, l'absence même de toute narrativité à l'exception d'un communiqué de presse qui met conjointement l'emphase sur la genèse de son mariage et de cette série, par ses références formelles à la statuaire antique, participe d'une mythification de sa propre famille déjà flagrante dans ses séries précédentes. L'artiste en a d'ailleurs fait l'aveu dans un entretien réalisé en 2007: «I want my work to be about people and places that I love, in all their complexity and the hope is that it will have a universal resonance in spite of being so personal. To that end, I'm not afraid to use lyricism, romance and intimacy, which offer terrible risk but also a ticket to transcendence ${ }^{16} »$. Pourtant, les références de l'artiste à l'Antiquité, tout comme son usage d'un procédé photographique pionnier évoquent peut-être ici un autre mythe. Un mythe qui remonte à la première moitié du 19e siècle, celui d'une Amérique antebellum, héroïque, progressiste et éprise de liberté, dont l'idéal démocratique était inspiré de l'Antiquité ${ }^{17}$. Si les poèmes de Walt Whitman ont porté cette exaltation à son expression culminante, la photographie - le daguerréotype, puis le tintype - a été l'instrument de cet idéal démocratique et de sa mise en scène. Si l'on veut bien considérer ce rapprochement, mais aussi l'attachement de la photographe à la nature et à son observation comme ferments d'une spiritualité, son approche de la matière, sa foi en la force de l'individu, Sally Mann semble s'inscrire dans la descendance d'un transcendantalisme américain où la nature - telle que définie par Emerson $^{18}$ - n'est certes plus le reflet du divin, à révérer en tant que telle, mais l'objet même d'une célébration toujours renouvelée. La nature de Sally Mann est celle de l'éternelle jeunesse ; elle est la grande ordinatrice, la mère terrible se nourrissant de la mort pour enfanter la vie en un cycle ininterrompu. Avec cette dernière série, Sally Mann affirme une nouvelle fois son statut de figure de proue au sein d'une veine de la photographie contemporaine volontiers anachronique dans son pictorialisme, décomplexée vis-à-vis de la modernité et revendiquant son archaïsme comme la marque de son esthétique et de ses préoccupations atemporelles.

\section{BIBLIOGRAPHIE}




\section{Photographies}

Fig. 1 Hephaestus, série Proud Flesh, tirage argentique d'après négatif sur plaque de verre au collodion humide, 38 x 34 cm, 2008, Gagosian Gallery, New York

Fig. 2 Was Ever Love, série Proud Flesh, tirage argentique d'après négatif sur plaque de verre au collodion humide, 38 x 34 cm, 2009, Gagosian Gallery, New York

Fig. 3 Ponder Heart, série Proud Flesh, tirage argentique d'après négatif sur plaque de verre au collodion humide, 38 x 34 cm, 2009, Gagosian Gallery, New York

Fig. 4 The Quality of the Affection, série Proud Flesh, tirage argentique d'après négatif sur plaque de verre au collodion humide, 38 x $34 \mathrm{~cm}, 2006$, Gagosian Gallery, New York

Fig. 5 Kingfisher's Wing, série Proud Flesh, tirage argentique d'après négatif sur plaque de verre au collodion humide, 38 x 34 cm, 2007, Gagosian Gallery, New York

Fig. 6 Memory's Truth, série Proud Flesh, tirage argentique d'après négatif sur plaque de verre au collodion humide, 38 x 34 cm, 2008, Gagosian Gallery, New York

\section{Ouvrages}

ARIES Philippe, Images de l'Homme devant la mort, Paris, Seuil, 1983

EMERSON Ralph Waldo, Nature, 1836

GREER Germaine, The Beautiful Boy, New York, Rizzoli, 2003

MANN Sally, Proud Flesh, Aperture, Gagosian Gallery, New York, 2009

MANN Sally, Deep South, Bulfinch Press, New York, 2005

MANN Sally, What Remains, Bulfinch Press, New York, 2003

MANN Sally, Mother Land: Recent Landscapes of Georgia and Virginia, Edwynn Houk Gallery, New York, 1997

MANN Sally, Still Time, Aperture, New York, 1994

MANN Sally, Immediate Family, Phaidon, London, 1992

MANN Sally, At Twelve, Portraits of Young Women, Aperture, New York, 1988

TRACHTENBERG Alan, In Romer Grant, Young America, The Daguerreotypes of Southworth \& Hawes, NY: Steidl, GEH, ICP, 2005 ("Daguerreotypy and Young America")

\section{Presse}

ALETTI Vince, "Family of Mann”, Critic's Notebook, 21 septembre, 2009

Gagosian Gallery, “Proud Flesh”, Press Release, Gagosian Gallery, New York, 23 juillet 2009, www.gagosian.com

MANN Sally, Proud Flesh, Artist's Statement, Gagosian Gallery, New York, 2009, www.gagosian.com PULVER Andrew, “Photographer Sally Mann's Best Shot”, The Guardian, 9 septembre 2009 


\section{Filmographie}

CANTOR Steven, What Remains, The Life and Work of Sally Mann, 2005, Zeitgeist Films

\section{NOTES}

1. «But even today, a woman is expected to take a narcissistic pleasure in fulfilling male fantasies rather than in exploring and acting out her own. There is a rigid distinction between the sex that looks and the sex that is looked at. The dichotomy is bound to breed perversion in both sexes, in the man voyeurism, hostility and envy, and in the woman masochism, exhibitionism and hypocrisy. Both men and women are impoverished »,Margaret Walters in Greer Germaine, The Beautiful Boy, New York, Rizzoli, 2003.

2. Dans son roman Lolita, Nabokov fait dire à Quilty: «A Frenchman once translated my Proud Flesh as La Fierté de la Chair. Absurd » Lolita, Penguin, 337). Il est possible que le titre de Sally Mann soit également une référence ironique à cette réplique, elle qui dans le recueil de Deep South (2005), parlait d'un « nabokovian state of grace ».

3. A ce sujet, voir l'étonnant documentaire intitulé What remains, The Life and Work of Sally Mann réalisé par Steven Cantor en 2005. Dans ce film, Steven Cantor s'est attaché à suivre la démarche de l'artiste tandis qu'elle préparait la série What remains, bien entendu, mais aussi Proud Flesh.

4. Voir l'article de Andrew Pulver "Photographer Sally Mann's best shot", The Guardian, 9 septembre 2009, disponible sur le site de la Galerie Gagosian.

5. Le communiqué de presse est également disponible sur le site internet de la Gagosian Gallery.

6. Proud Flesh, Artist's statement, à lire sur le site internet de la Gagosian Gallery.

7. Voir la série Last Measure du recueil Deep South (2005) ou la série Antietam incluse dans le recueil What remains publié en 2003.

8. C'est dans ce centre médico-légal que Sally Mann a mené son enquête sur la décomposition du corps humain, enquête dont elle a tiré une série baptisée Matter Lent.

9. Mann Sally, Immediate Family, Aperture, New York, 1992.

10. Mann Sally, Proud Flesh, Aperture, Gagosian Gallery, New York, 2009.

11. Notons que Carolyn $\mathrm{D}$. Wright, poète, traductrice et éditrice américaine, avait déjà collaboré à un projet photographique avec Deborah Luster, artiste qui a elle aussi recours à un procédé historique dans sa pratique photographique, le tintype. Cette collaboration a abouti sous la forme d'un livre intitulé One Big Self: Prisoners of Louisiana, 2003 (Twin Palms). Carolyn D. Wright est par ailleurs l'épouse du poète Forrest Gander, qui en 2005, a publié Eye Against Eye (New Directions), un recueil de poèmes mis en relation avec quelques unes des photographies de la série Deep South de Sally Mann.

12. Au sujet de l'exposition Faces, qui a eu lieu à Paris, au sein de la galerie Karsten Greve, du 15 novembre 2007 au 19 janvier 2008, voir la critique de Guillemette Minisclou, «Le Mausolée de Sally Mann », Transatlantica [En ligne], 1 | 2009, mis en ligne le 20 juillet 2009, Consulté le 28 octobre 2009. URL: http://transatlantica.revues.org/index4311.html

L'article présente en outre un certain nombre de photographies incluses dans le recueil What Remains (Bulfinch Press, 2003), dont les séries Antietam et Matter Lent font partie.

13. Proud Flesh, Artist's Statement, Gagosian Gallery

14. Artist's statement

15. À ce sujet, voir les différents textes, de la main de l'artiste, du recueil Deep South (2005).

16. In «Sally Mann», Festival of the Photograph, Charlottesville, 2007, http:// www.festivalofthephotograph.org/look3/mann.html. 
17. À ce propos, voir le texte d'Alan Trachtenberg, "The Daguerreotype and Antebellum America » inclus dans Romer Grant, Wallis Brian, Young America, The Daguerreotypes of Southworth \& Hawes, NY, Steidl, Georges Eastman House, International Center of Photography, 2005.

18. "Philosophically considered, the universe is composed of Nature and the Soul. Strictly speaking, therefore, all that is separate from us, all which Philosophy distinguishes as the NOT ME, that is, both nature and art, all other men and my own body, must be ranked under this name, NATURE », Emerson, Nature, introduction, 1836.

INDEX

Thèmes : Trans'Arts

\section{AUTEUR}

\section{LISA ROSS}

Université Paris VII - Denis Diderot, Institut d'études anglophones, Charles VDoctorante en études américaines 